\title{
Les collections de l'ancienne Bibliothèque nationale de Prusse à la Bibliothèque Jagellonne de Cracovie
}

\section{Zdzisław Pietrzyk}

Traducteur : Aude Therstappen

\section{(2) OpenEdition}

Journals

Édition électronique

URL : https://journals.openedition.org/rbnu/2848

DOI : $10.4000 /$ rbnu. 2848

ISSN : 2679-6104

\section{Éditeur}

Bibliothèque nationale et universitaire de Strasbourg

\section{Édition imprimée}

Date de publication : 1 mai 2012

Pagination : 54-65

ISSN : 2109-2761

\section{Référence électronique}

Zdzisław Pietrzyk, « Les collections de l'ancienne Bibliothèque nationale de Prusse à la Bibliothèque Jagellonne de Cracovie », La Revue de la BNU [En ligne], 5 | 2012, mis en ligne le 01 mai 2012, consulté le 25 août 2021. URL : http://journals.openedition.org/rbnu/2848 ; DOI : https://doi.org/10.4000/rbnu. 2848

Ce document a été généré automatiquement le 25 août 2021.

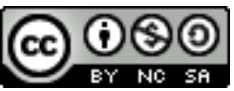

La Revue de la BNU est mise à disposition selon les termes de la Licence Creative Commons Attribution - Pas d'Utilisation Commerciale - Partage dans les Mêmes Conditions 4.0 International. 


\title{
Les collections de l'ancienne Bibliothèque nationale de Prusse à la Bibliothèque Jagellonne de Cracovie
}

\author{
Zdzisław Pietrzyk \\ Traduction : Aude Therstappen
}

La Bibliothèque Jagellonne est la plus ancienne bibliothèque universitaire de Pologne. Elle est probablement née en même temps que l'université de Cracovie, fondée par le roi de Pologne Casimir le Grand le 12 mai 1364. Les premiers documents qui citent la bibliothèque universitaire datent de l'année 1403. Jusqu'à la fin du $18^{\mathrm{e}}$ siècle, il n'existait pas de bâtiment principal mais des bibliothèques de facultés et de collèges. La plus grande et la plus précieuse collection était celle du Collegium Maius. Les fonds de la bibliothèque universitaire se développèrent grâce aux dons, aux legs et aux fondations de ses bienfaiteurs, et dès le tournant des $15^{\mathrm{e}}-16^{\mathrm{e}}$ siècles se constituèrent des collections de manuscrits et d'incunables légués à la bibliothèque par les professeurs de l'université. Le $16^{e}$ et la première moitié du $17^{e}$ siècle constituent une période faste pour le développement de la bibliothèque universitaire de Cracovie puisque les fondations et notamment celles de Tomasz Obiedziński et de Benedykt de Koźmin apportèrent progressivement d'importants moyens financiers. Les professeurs et les anciens étudiants offraient leurs collections rares et précieuses à leur université qui portait à l'époque le nom d'Académie de Cracovie, comme par exemple l'évêque de Cracovie Piotr Tomicki (1464-1537), l'évêque de Plock Piotr Wolski (1531-1590) ou encore Jan Brożek (1585-1652). En revanche, la seconde moitié du $17^{\mathrm{e}}$ siècle, marquée par les attaques suédoises des années 1655-1657 et 1702-1705 ainsi que par les épidémies fréquentes à Cracovie, qui accompagnèrent le déclin politique et économique de l'État, concourut au recul de l'Académie. De 1777 à 1780, Hugo Kołłątaj réforma l'université, ce qui eut entre autres comme conséquence la fondation d'une bibliothèque centrale, abritée dans le bâtiment médiéval du Collegium Maius. La fin du $18^{\mathrm{e}}$ et le début du $19^{\mathrm{e}}$ siècle virent à nouveau un enrichissement des collections de la 
bibliothèque universitaire de Cracovie, désormais baptisée "Bibliothèque Jagellonne ». Des bibliothèques privées, qui comptaient souvent plus de 1000 titres, furent léguées entre autres par l'évêque Adam Grabowski, Józef Bogucicki, Sebastian Sierakowski, Józefa Gostowska ainsi que par les directeurs de bibliothèque Jacek Przybylski et Jerzy Samuel Bandtkie.

Grâce au travail d'excellents bibliothécaires tels que Jerzy Samuel Bandtkie, Józef Muczkowski et Karol Estreicher qui dirigèrent successivement la Bibliothèque Jagellonne, cette institution devint la plus grande bibliothèque polonaise à l'époque (1795-1918) de la perte d'indépendance du pays. Après qu'il eut recouvert sa souveraineté, il devint urgent de répondre aux besoins scientifiques et culturels de la population et de sauvegarder l'héritage culturel de l'État pluriethnique de la Première République. Dans les années 1931-1939, un nouveau bâtiment fut construit pour la Bibliothèque Jagellonne, à l'époque un des plus modernes d'Europe. Et c'est pendant l'occupation nazie que fut organisé le déménagement des collections. L'inauguration de la nouvelle bibliothèque par les autorités national-socialistes eut lieu le 6 avril 1941. Globalement, la Pologne a perdu plus de la moitié de son patrimoine livresque pendant l'occupation nazie et il faut souligner que la perte des collections les plus précieuses de la Bibliothèque nationale et des Archives de documents historiques fut provoquée par un "commando incendiaire " qui déclencha délibérément un incendie en octobrenovembre 1944 après la défaite de l'insurrection de Varsovie. Il n'est possible ni d'évaluer les pertes subies dans les collections des bibliothèques polonaises ni de les réparer. Des manuscrits et des imprimés anciens d'une valeur inestimable partirent en fumée tout comme des centaines de milliers de livres.

Fragment tiré des Gladiatoria, $15^{\mathrm{e}}$ siècle

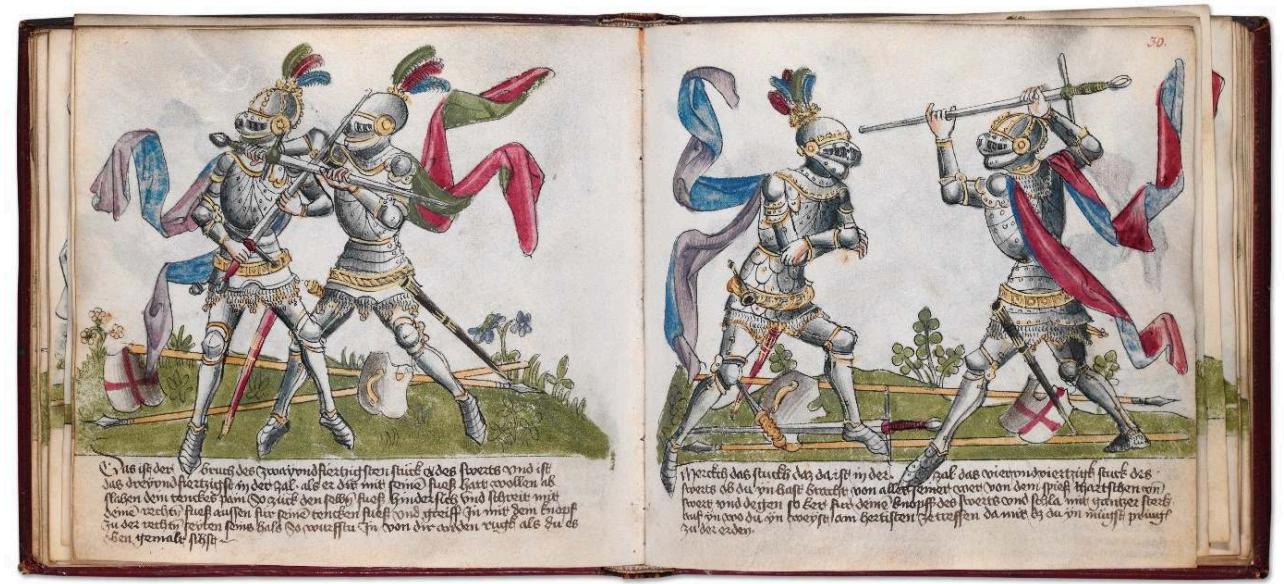

Clichés Bibliothèque Jagellonne

3 Cependant, la Bibliothèque Jagellonne fait partie des très rares établissements polonais à n'avoir subi que peu de pertes pendant la Seconde Guerre mondiale et elle était, à la fin du conflit, l'une des rares à disposer d'un bâtiment neuf et d'importantes surfaces de magasins. La guerre provoqua non seulement d'immenses pertes humaines mais également de grands mouvements de population, conséquences des poursuites et de la terreur nazies. Les changements politiques qui intervinrent en Pologne après 1945 avec la prise de pouvoir des communistes entraînèrent également le départ des nobles et des couches les plus aisées de la population. Beaucoup de bibliothèques privées, parfois 
petites mais précieuses, furent abandonnées à l'occasion de cette migration. Le ministère polonais de la Culture créa d'ailleurs en 1945 un service pour la sauvegarde de ces collections et cette institution autonome utilisa en priorité les grandes caves de la Bibliothèque Jagellonne. Des livres venus de toute la Pologne furent ainsi rassemblés entre 1945 et 1947 dans le nouveau bâtiment de la bibliothèque. Après que ces collections eurent été classées, elles furent envoyées, sans souci des droits de propriété, dans les villes dont les bibliothèques avaient subi les plus grandes pertes pendant la guerre. Ainsi différentes collections issues de toute la Pologne ont-elles trouvé un asile temporaire entre les murs de la Bibliothèque Jagellonne.

4 Mais c'est aussi vers cette bibliothèque qu'ont été acheminés les livres qui se sont retrouvés, après la fin de la guerre, à l'intérieur des frontières polonaises, comme ce fut le cas pour une partie des collections de la Bibliothèque nationale de Prusse.

Fondée en 1661 par Frédéric Guillaume de Brandebourg sous le nom de Bibliothèque princière de Cölln an der Spree, elle changea de nom en 1701 pour devenir Bibliothèque royale de Berlin et, après l'abrogation de la monarchie prussienne et de l'Empire allemand en 1918, elle fut rebaptisée Bibliothèque nationale prussienne de Berlin. Cet établissement, le plus grand d'Allemagne, devint particulièrement célèbre grâce à l'acquisition de fonds et de collections de savants et d'artistes renommés. Les rois de Prusse contribuèrent également activement à l'accroissement de ses collections qui se développèrent rapidement à partir de la fin $\mathrm{du} 17^{\mathrm{e}}$ siècle. Au $19^{\mathrm{e}}$ siècle, ses fonds étaient déjà devenus inestimables grâce à l'acquisition de bibliothèques comme celles de Friedrich Nicolai, Johann C. Adelung, Wilhelm von Humboldt et Karl A. Varnhagen. La bibliothèque comptait au début de la Première Guerre mondiale 1,5 million de documents. En mars 1942, ses collections s'élevaient à plus de 3 millions de volumes. Les attaques aériennes des forces britanniques sur Berlin touchèrent aussi le bâtiment de la bibliothèque. Les premières bombes tombèrent le 10 avril 1941 et c'est peu après qu'il fut décidé de stocker les collections les plus précieuses en dehors de Berlin. En août et septembre 1941, les lieux de stockage furent choisis : le château de Banz près de Bamberg, le couvent bénédictin de Beuron près de Sigmaringen et le château Fürstenstein (Książ en polonais) près de Waldenburg (Wałbrzych en polonais), en basse Silésie $^{1}$. Les collections de la Bibliothèque nationale de Prusse furent ainsi stockées pendant les années 1941-1944 dans vingt-neuf localités différentes sur le territoire du Troisième Reich. Pas moins de 3 millions de volumes ont été ainsi transportés en dehors de Berlin. Les premiers transports de livres en direction de Fürstenstein commencèrent à la fin du mois d'octobre et au début du mois de novembre 1941. Comme ce château avait finalement été choisi pour être l'un des quartiers militaires d'Hitler, les livres furent en définitive envoyés à Grüssau (en polonais Krzeszów) en deux étapes, en 1941 puis en $1943^{2}$. Dans le couvent bénédictin de Grüssau, renommé Krasnobór juste après la guerre, furent ainsi transportées 505 caisses de livres. 


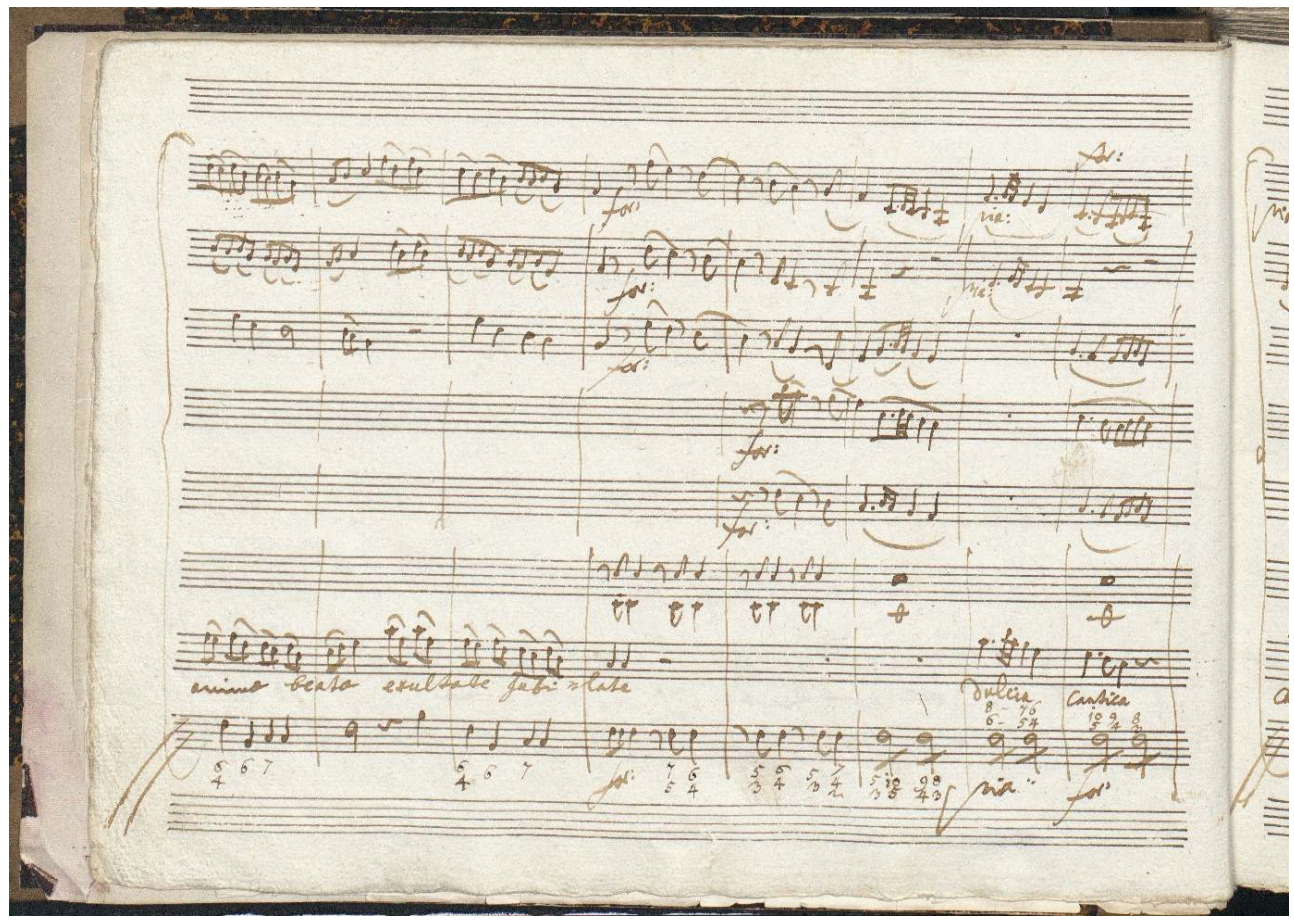

Clichés Bibliothèque Jagellonne

6 Après la fin de la Seconde Guerre mondiale, la Silésie se trouvait en territoire polonais et à la fin du mois d'août 1945, la milice polonaise prit possession du couvent. Les collections de l'ancienne Bibliothèque nationale de Prusse furent envoyées à Cracovie le 12 mai 1946 sous la direction d'un groupe de bibliothécaires polonais appartenant au service de la sauvegarde des collections du ministère de la Culture, accompagnés par le Dr. Stanisław Sierotwiński. Le destin de ces collections allait à présent devenir secret. On savait seulement qu'elles avaient été récupérées par la Pologne. Certains craignirent que cette partie des collections de la bibliothèque berlinoise n'ait été détruite pendant la guerre ou pendant les premières années qui suivirent la fin du conflit. De fait, après avoir été transportés de Grüssau à Cracovie, les livres furent stockés pendant plus d'un an dans des couvents de missionnaires et de dominicains avant que leur transfert vers la Bibliothèque Jagellonne ne soit décidé pour des raisons de conservation. Arrivées en octobre 1947, les caisses furent déballées le $1^{\text {er }}$ novembre 1947 et leur contenu examiné et classé jusqu'au $1^{\text {er }}$ février 1948 .

7 Les fonds en provenance de Berlin et stockés à Grüssau étaient empaquetés dans des caisses et divisés en deux catégories. Dans la première se trouvaient les manuscrits, autographes, incunables, documents sur l'Orient ainsi que les documents rares et les imprimés d'art. La seconde rassemblait les imprimés sur le judaïsme, la collection de guerre, la philologie, les imprimés italiens, les périodiques, les imprimés slaves et les atlas. Mais le "trésor prussien» entreposé dans le couvent de Grüssau comptait 505 caisses, or seules 490 arrivèrent à la Bibliothèque Jagellonne et il n'y avait pas de liste du contenu de chaque caisse. De plus, certains manuscrits étaient humides et abîmés. Parmi les collections, ce sont les autographes, les manuscrits grecs et orientaux qui ont le plus souffert de l'humidité. Mais d'autres documents en provenance de Silésie 
vinrent aussi rejoindre le bâtiment de la Bibliothèque Jagellonne, et en particulier des périodiques et des imprimés récents qui n'étaient ni empaquetés ni inventoriés.

En 1957, on recommanda à Jan Baumgart, le directeur de la bibliothèque, de préparer ces collections en vue de leur retour à Berlin. On acheta alors des appareils pour réaliser des copies sur microfiches des documents les plus précieux mais peu de temps après, le ministère de l'Enseignement supérieur revint sur sa décision. Quelques années plus tard, il fut décidé de renvoyer une masse colossale de périodiques en Allemagne. Plus de dix wagons remplis de périodiques partirent de Łódź en direction de l'ancienne Bibliothèque nationale de Prusse à Berlin, où l'administration de la RDA avait fondé la nouvelle Bibliothèque nationale. Toutefois, les collections les plus précieuses restèrent à la Bibliothèque Jagellonne. Entre-temps, différents experts en droit international avaient été consultés afin de statuer sur la propriété de ces fonds. Tous furent unanimes pour déclarer que les collections trouvées à Grüssau étaient à présent la propriété de l'État polonais, mais la présence de ces livres au sein des collections de la Bibliothèque Jagellonne demeurait encore largement ignorée du grand public. Ce n'est qu'au milieu des années 1970 que les premiers préparatifs commencèrent pour intégrer la collection «Berlinka » aux fonds de la bibliothèque. Mais là encore, cette décision allait être brusquement révoquée et la question de l'avenir de ces fonds aussi bien que celle de leur disponibilité allaient être remises à plus tard. En 1977, un autre événement souligne l'inconséquence politique de l'administration par rapport à la collection berlinoise. Lors de sa visite à Berlin le 29 mai 1977, le Premier secrétaire du Parti des travailleurs polonais, Edward Gierek, remit au secrétaire général du SED (le parti communiste de la RDA) les autographes de la $9^{e}$ symphonie et du $3^{e}$ concerto pour piano de Beethoven ainsi que les manuscrits de La flûte enchantée, de la Messe en ut mineur et de la Symphonie Jupiter de Mozart. Les manuscrits du Concerto en ut mineur et de la $3^{e}$ sonate pour violon seul de Johann Sebastian Bach faisaient aussi partie du cadeau.

En juillet 1977, la Bibliothèque nationale de Berlin transmit une copie des catalogues et des inventaires des titres et des cotes des documents envoyés pendant la guerre à Grüssau. Finalement, ce n'est qu'en 1979 que la collection «Berlinka » fut accessible aux chercheurs dûment munis d'une autorisation spéciale du ministère. Avec l'arrivée de Józef Andrzej Gierowski à la tête de l'Université Jagellonne et de Jan Pirożyński à la tête de la bibliothèque, il devint possible d'assurer une communication "normale » de cette collection, consultable par les chercheurs depuis 1981 aussi bien sur place dans les salles de la bibliothèque que sur microfilm. Les collections conservées à la Bibliothèque Jagellonne sont en grande partie classées au patrimoine mondial de l'UNESCO. 


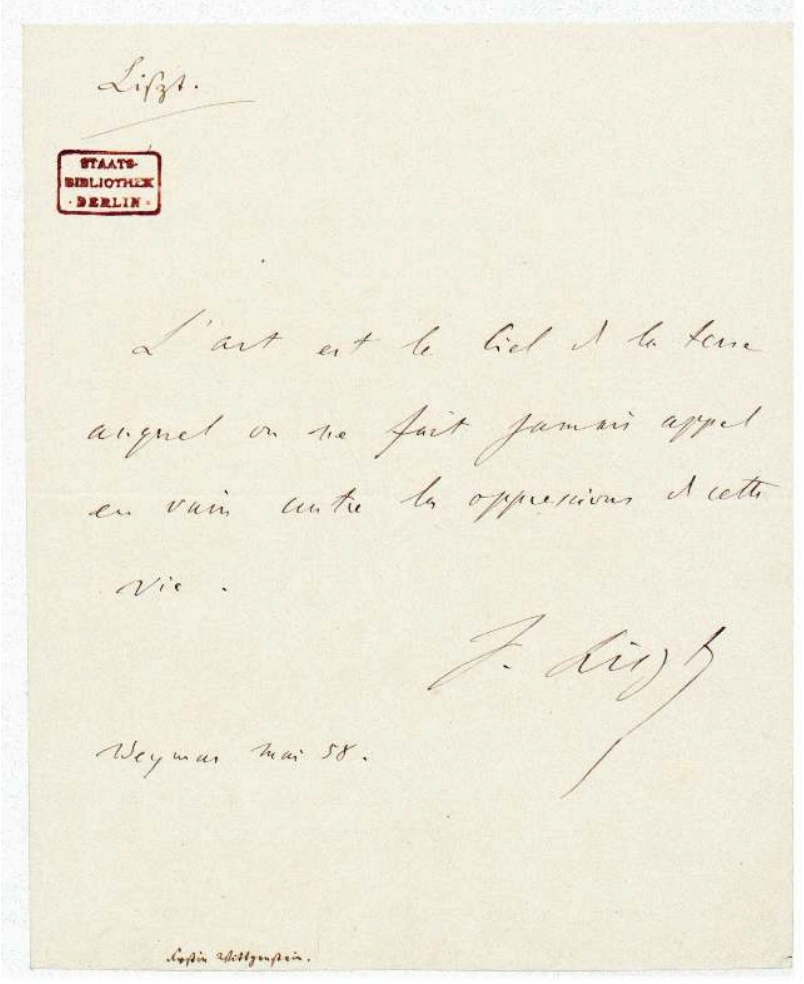

Clichés Bibliothèque Jagellonne

10 L'un des premiers ensembles accessibles aux chercheurs est celui des Libri picturati, qui constituent un véritable monument d'art manuscrit et graphique, incontournable pour la culture, la faune et la flore du Brésil. Les Libri picturati se composent entre autres de quatre volumes avec des gouaches et des dessins à l'aquarelle qui illustrent l'expédition brésilienne du comte Johann Moritz von Nassau-Siegen. Ces ouvrages furent acquis en 1668 par l'Électeur Frédéric Guillaume. L'autre ouvrage emblématique de cet ensemble est le Livre des tournois. Depuis quelques années, la bibliothèque a commencé à restaurer les "volumes brésiliens" grâce au concours du ministère de la Culture. Cette restauration, longue et minutieuse, est encore en cours. Un ouvrage en plusieurs volumes reprenant un grand nombre d'illustrations des Libri picturati est entretemps paru au Brésil. Un groupe de chercheurs internationaux, comprenant notamment des botanistes et des historiens d'art, travaille sur cette collection et une exposition présentée en 2002 à la Bibliothèque Jagellonne a contribué à faire connaître ce trésor à un large public.

11 Mais les chercheurs sont également très intéressés par les collections musicales. Les fonds musicaux de la Bibliothèque Jagellonne comptent plus de 9000 volumes : 8658 imprimés des $16^{\mathrm{e}}$ et $17^{\mathrm{e}}$ siècles et 524 manuscrits. On y trouve les œuvres de compositeurs italiens, allemands, français et polonais ainsi que des imprimés précieux de Venise, Rome et Florence puisque c'est à Florence que les opéras des compositeurs de la Camerata florentina étaient publiés. Aleksandra Patalas a publié en 1999 un catalogue des collections musicales issues de l'ancienne Bibliothèque nationale de Prusse et conservées à la Bibliothèque Jagellonne et le personnel de la bibliothèque s'est beaucoup investi dans ce travail. La deuxième partie des fonds de musique se compose de manuscrits et repose sur une collection de grands maîtres, constituée au 
$19^{\mathrm{e}}$ siècle. 100 manuscrits musicaux du moyen-âge et 400 autographes de grands compositeurs tels que Buxtehude, Bach et ses fils, Telemann, Haydn, Mozart, Beethoven, Cherubini, Mendelssohn-Bartholdy, Schubert, Schumann, Meyerbeer, Paganini, Loewe, Brahms, Busoni et bien d'autres encore en font partie. La Bibliothèque Jagellonne a souvent mis cette collection à la disposition des chercheurs, mais refuse la consultation des autographes de grands compositeurs aux personnes pour lesquelles elle constitue de toute évidence plus une attraction touristique qu'un objet d'étude. Elle mène également depuis 1999 un projet d'édition sur microfiches des autographes des grands compositeurs, en partenariat avec la Bibliothèque nationale de Berlin.

Les œuvres de Johann Sebastian Bach et de ses fils, de Beethoven et de Mozart sont déjà parues sous cette forme. Depuis juin 2010, les autographes de Bach ainsi que les copies d'œuvres d'autres compositeurs réalisées de sa main ont été rassemblés virtuellement sur le site www.bach-digital.de.

Un autre projet, de 2006 à 2009, a concerné la publication par The Packard Humanities Institute des opéras de Mozart en fac-similés. Par ailleurs, à l'occasion du $170^{\mathrm{e}}$ anniversaire de sa mort, l'année 1997 était « l'année Beethoven » et la première édition du Festival d'opéra Ludwig van Beethoven a été lancée à Cracovie, accompagnée d'une exposition des manuscrits et autographes musicaux de la Bibliothèque Jagellonne. Depuis cette date, certains des trésors de la collection musicale sont exposés chaque année à la bibliothèque au moment du Festival Beethoven. C'est aussi une occasion unique pour le grand public de voir les manuscrits des plus grands compositeurs dont la consultation est, le reste du temps, réservée aux chercheurs. Il y a d'ores et déjà eu quinze expositions de manuscrits musicaux et ce rendez-vous annuel constitue un événement attendu et fédérateur aussi bien pour les mélomanes que pour la Bibliothèque Jagellonne. Il convient d'ailleurs de souligner ici l'excellente coopération avec la Bibliothèque nationale de Berlin puisque, pour la première exposition, des autographes de Beethoven, conservés à Berlin, avaient été prêtés afin de reconstituer l'ensemble de l'œuvre de ce compositeur.

Une partie de la collection de manuscrits de l'ancienne Bibliothèque nationale de Prusse est également conservée dans les fonds de la Bibliothèque Jagellonne. Il s'agit des «manuscrits reliés » qui rassemblent une collection de généalogie, six legs et deux collections d'autographes. A l'origine, cet ensemble était classé par langue. Ainsi on peut dénombrer 230 volumes de manuscrits français allant $d u 13^{e}$ au $19^{e}$ siècle. Les thèmes abordés sont variés et vont de la littérature en ancien français aux récits de voyages des $18^{\mathrm{e}}$ et $19^{\mathrm{e}}$ siècles, des documents relatifs aux guerres napoléoniennes à l'histoire des églises réformées en passant par les livres de cuisine. En 2007 est paru un catalogue des manuscrits en ancien français, toujours en collaboration avec le département des manuscrits de la Bibliothèque nationale de Berlin. On en trouvera les références en fin d'article. Tous ces manuscrits constituent des sources précieuses pour l'histoire du moyen-âge en France. Aujourd'hui, Piotr Tylus et son équipe de chercheurs romanistes de l'Université de Cracovie ont fini l'inventaire de tous les manuscrits en langues romanes et le résultat de leurs recherches a fait l'objet de diverses publications et d'articles dans la revue Fibula. 


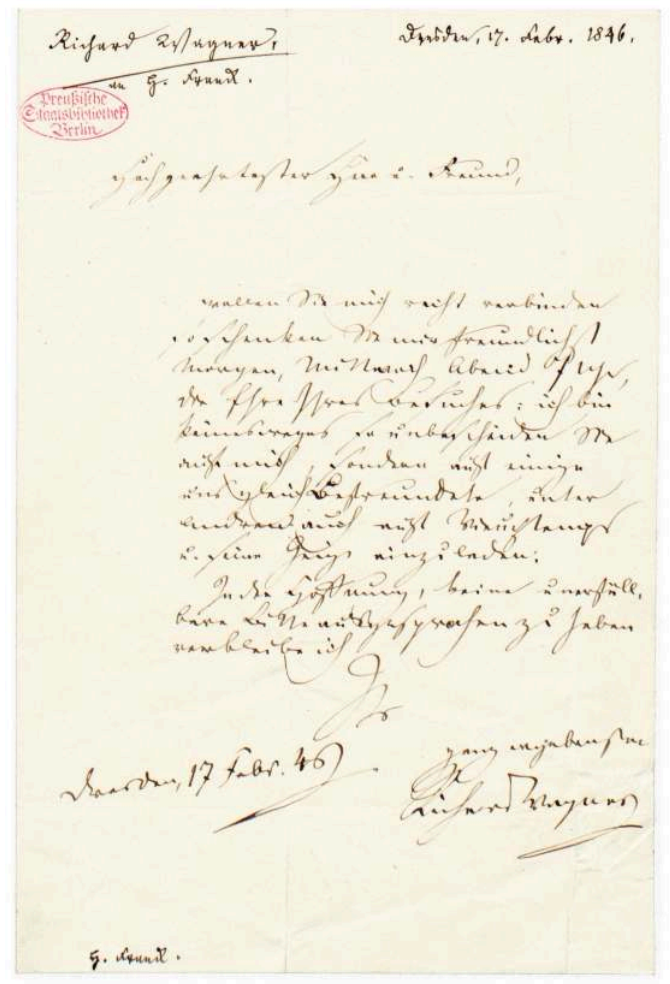

Clichés Bibliothèque Jagellonne italiens sont conservés dans 136 volumes qui contiennent des textes de littérature médiévale italienne, des chroniques de villes, des documents généalogiques ou des écrits politiques. Les manuscrits espagnols représentent 116 volumes et contiennent notamment des textes en langue catalane, des œuvres classiques de la littérature espagnole et catalane, des documents sur les guerres napoléoniennes, sur le commerce espagnol ainsi que sur l'histoire des colonies espagnoles en Amérique du sud, particulièrement au Chili. La collection de manuscrits grecs (138 volumes), latins (67 volumes) ou slaves est également significative.

Les manuscrits allemands représentent 78 volumes. Les plus anciens datent du $10^{\mathrm{e}}$ siècle et ils sont de grande valeur pour l'étude de la langue allemande médiévale. Les manucrits les plus souvent étudiés et cités par les chercheurs sont les textes des Nibelungen, la Chronique du monde de Rudolf von Ems, Tristan et Gauvain d'Einhard von oberge, sans oublier Wigelois et Athis et Prophilias. Parmi les autographes les plus précieux, on compte également ceux de Martin Luther, des lettres de Goethe et le journal de Rainer Maria Rilke.

Un fonds particulier est celui des livres d'amitié ou alba amicorum, qui représentent 89 volumes allant $\mathrm{du} 16^{\mathrm{e}}$ au $20^{\mathrm{e}}$ siècle. La plupart viennent d'Allemagne mais certains appartiennent aussi à des étudiants silésiens du tournant du $16^{\mathrm{e}}$ et du $17^{\mathrm{e}}$ siècle. On y trouve aussi des inscriptions d'étudiants polonais qui étudièrent dans des universités étrangères comme par exemple les frères Wacław et Rafał Leszczyński (1594), Mikołaj Ostroróg (1582), Stanisław Buczyński (1589), Salomon Rysiński Panterus (1589) et Marcin Ruar (1610). Cette collection a fait l'objet d'une thèse de doctorat d'Aleksandra 
Golik-Prus, qui porte plus particulièrement sur les inscriptions latines de la fin du $16^{\mathrm{e}}$ et du début du $17^{\mathrm{e}}$ siècle.

La collection de généalogie est également particulièrement précieuse et tout spécialement les ouvrages ayant appartenu à König. 187 volumes contiennent des copies de documents, des tableaux généalogiques et un grand nombre de documents rares concernant plus de 3000 familles nobles originaires d'Allemagne, de Poméranie, de la Grande Pologne et de Prusse. On y trouve aussi des renseignements sur de grandes familles polonaises nobles, comme les Bażyński, les Czapski et les Działyński. Cette collection généalogique est très souvent utilisée par les chercheurs aussi bien sur place que par le biais de demandes de reproductions. Mais il existe aussi de plus petits fonds de manuscrits comme le fonds portugais (3 volumes) et le fonds américain (15 volumes) qui sont en partie composés de fac-similés du Codex de Dresde, du Codex Perez Maya Tzental et du Codex Nuttal. Une dizaine de manuscrits originaux datant de l'époque de la conquête de l'Amérique complètent ce fonds.

Tout cet ensemble compose les "Collectanea linguistica» qui rassemblent des documents précieux pour la recherche en linguistique et l'étude des langues exotiques. Ils proviennent des collections de Jakob Michael Reinhold Lenz (1750-1792), Alexander (1769-1859) et Wilhelm von Humboldt (1767-1835). D'autres grands savants ont également légué leurs collections, conservées à la Bibliothèque Jagellonne. On peut citer Gustav Freytag (1816-1895), historien, écrivain et professeur à l'université de Breslau mais aussi August Heinrich Hoffmann von Fallersleben (1798-1874), germaniste, poète et également professeur de cette université. Il faut toutefois noter qu'il manque malheureusement à son legs l'autographe de l'hymne national allemand qui était, à l'époque de la guerre, emprunté et donc absent des fonds de la Bibliothèque nationale de Prusse. Citons aussi le legs de Georg Schweinfurt (1836-1925), spécialiste des études africaines. Ce fonds permet une plongée dans l'histoire du colonialisme allemand et rassemble un grand nombre de documents rares et de correspondances.

Plus de 500 boîtes contiennent aussi de grandes collections d'autographes : celle de Karl August Varnhagen von Ense (1785-1858) rassemble des documents uniques sur la vie littéraire et politique en Allemagne et en Europe à la fin du $18^{\mathrm{e}}$ et au début du $19^{\mathrm{e}}$ siècle. Diplomate, écrivain et critique littéraire, Varnhagen et sa femme Rahel Levin (1771-1833) occupaient une place privilégiée dans les milieux littéraires et politiques berlinois. Le salon de Rahel Varnhagen était un des centres intellectuels de Berlin et les deux époux entretenaient un grand réseau de correspondances internationales. Cette collection d'autographes, de lettres, de journaux intimes et de notes compte également plus de 9000 textes littéraires ainsi que des imprimés, des coupures de presse et des illustrations. Elle constitue une source exceptionnelle sur l'histoire de la Prusse dans la première moitié du $19^{\mathrm{e}}$ siècle ainsi que sur l'émancipation des Juifs à cette époque. 
Enluminure tirée du Liber hymnorum de Notker Balbulus, $9^{\mathrm{e}}$ siècle

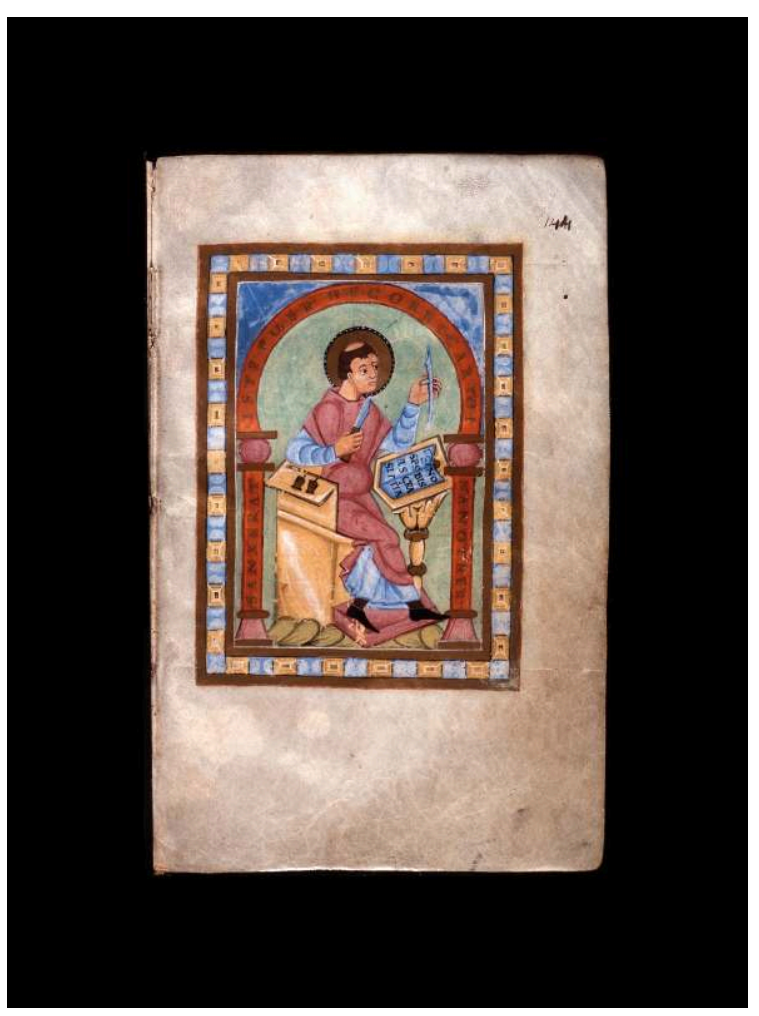

Clichés Bibliothèque Jagellonne

21 En plus des papiers d'August et de Rahel Varnhagen, cette collection contient aussi trois fonds distincts : les archives du comte Hermann von Pückler-Muskau (1785-1881), le legs de Ludmilla Assing (1821-1880) et la correspondance de Jean Henri Samuel Formey (17111791), président de l'Académie royale des sciences de Prusse. Cette collection est connue depuis plus de quatre-vingt-dix ans grâce au catalogue édité par Ludwig Stern. Cet ensemble d'autographes rassemble un fonds particulièrement important de manuscrits sur feuillets mobiles. Il s'agit de lettres, de manuscrits littéraires, de fragments d'œuvres scientifiques et d'inscriptions dans des livres d'amitié. On y trouve des manuscrits de personnages issus des mondes scientifique, culturel, littéraire ou politique, venant des cours royales et princières de toute l'Europe depuis la fin du $15^{\mathrm{e}}$ siècle jusqu'en 1939. C'est dans ce fonds que l'on trouve les correspondances de Formey, des frères Grimm, de Hegel, Herder, Schelling et de Jean Paul Richter. Il est aussi riche d'autographes de Luther, Melanchthon, Calvin et également de grands représentants de l'Eglise catholique.

La collection Varnhagen et les autres ensembles d'autographes sont les fonds les plus souvent consultés de la bibliothèque. Durant les vingt dernières années ils ont fait l'objet de nombreuses publications: correspondance de Rahel Varnhagen, Jean Paul Richter et J. H. S. Formey, documents des fonds de Lenz et de Wilhelm von Humboldt. Jutta Weber, de la Bibliothèque nationale de Berlin, a d'ailleurs réalisé et édité un index de cette collection.

Les manuscrits de l'ancienne Bibliothèque nationale de Prusse sont aussi montrés à un plus large public à l'occasion d'expositions consacrées aux grands représentants de la littérature allemande. Ainsi, en 2006, la Bibliothèque Jagellonne a organisé une exposition sur l'œuvre des frères Grimm, en coopération avec le Musée Grimm de 
Kassel et en $2011 \mathrm{a}$ eu lieu une exposition dédiée à Heinrich von Kleist, en partenariat avec l'Institut d'études germaniques de l'Université de Cracovie et l'Institut pour la langue allemande de l'Université de Cologne.

Dernier grand pan des fonds de l'ancienne Bibliothèque nationale de Prusse, les imprimés composent un ensemble de plus de 12000 volumes qui comporte notamment des incunables (le plus précieux est un recueil de 1488 de l'imprimeur de Leipzig Mauritius Barndis). Les "Aldines» (194 volumes) forment également un ensemble précieux qui provient de l'imprimeur vénitien Alde Manuce et de ses successeurs. On y trouve aussi des ouvrages imprimés dans d'autres villes italiennes ainsi qu'à Paris et à Lyon, et qui se rattachent à l'art typographique de Manuce.

La littérature allemande du $16^{\mathrm{e}}$ jusqu'au $18^{\mathrm{e}}$ siècle est la plus représentée dans ce fonds avec les œuvres de Hans Sachs, Sebastian Brandt, Ulrich Hutten, Andreas Gryphius, Johann Wolfgang Goethe, Gotthold Ephraim Lessing et Friedrich Schiller. Il existe également plus de 5000 " écrits de circonstance " qui datent en majorité du $17^{\mathrm{e}}$ siècle, réalisés à l'occasion d'un mariage, d'un décès ou d'une naissance et qui sont d'autant plus intéressants qu'ils proviennent de familles originaires de villes aujourd'hui polonaises comme Gdańsk (Danzig), Toruń (Thorn) et Elbląg (Elbing). Ces documents datent de la période de la guerre de Trente Ans et de la guerre entre la Suède et la Pologne et constituent une source importante pour l'histoire de la transmission des idées à cette époque. Les récits de voyages de cette période sont également très intéressants, ainsi que plusieurs fonds numériquement moins importants: une centaine de livres sur le judaïsme, inventoriés dans le catalogue réalisé par Jerzy Pilarczyk de l'Université Jagellonne, ou encore la collection des périodiques savants de la seconde moitié du $17^{\mathrm{e}}$ siècle, la collection de cartes, les manucrits et les textes orientaux (chinois, coréens, arabes et syriens) et des textes en langues rares qui suscitent peu l'intérêt des chercheurs à l'exception des manuscrits et imprimés tibétains dont un catalogue vient d'être édité par des chercheurs de l'Université de Varsovie.

Enfin, plus de 50000 imprimés modernes, édités après 1800, sont également conservés dans le fonds de la Bibliothèque Jagellonne et se trouvent à la disposition du public. Tout cet ensemble de grande valeur, provenant de l'ancienne Bibliothèque nationale de Prusse, est bien sûr l'objet d'importants efforts de conservation et de restauration que la Bibliothèque Jagellonne mène au fil du temps.

\section{BIBLIOGRAPHIE}

M. Mejor, A. Helman-Ważny, T. Kunga Chashab, A Preliminary Report on the Wanli Kanjur Kept in the Jagiellonian Library, Cracovie, Varsovie, 2010

A. Patalas, Catalogue of early music prints from the collections of the former Preußische Staatsbibliothek in Berlin, kept at the Jagiellonian Library in Cracow, Cracovie, 1999 
Z. Pietrzyk, « Zbiory z byłej Pruskiej Biblioteki Państwowej w Bibliotece Jagiellońskiej », in Alma Mater, 2008, n 100, pp. 15-19

K. Pilarczyk, Katalog Judaików - starych druków w zbiorach Biblioteki Jagiellońskiej w Krakowie z dawnej Pruskiej Biblioteki Państwowej w Berlinie, z faksymilami wybranych elementów opisanych druków,

Cracovie, 2011

W. Schochow, Bücherschicksale. Die Verlagerungsgeschichte der Preußischen Staatsbibliothek, Berlin New York, 2003

R. Sosnowski, P. Tylus, Co mówią stare rĘkopisy, Cracovie, 2010

D. Stutzmann, P. Tylus, Manuscrits médiévaux français et occitans de la Preussische Staatsbibliothek et de la Staatsbibliothek zu Berlin Preussischer Kulturbesitz, Wiesbaden, 2007

P. Tylus, Manuscrits français de la collection berlinoise disponibles à la Bibliothèque Jagellonne de Cracovie (XVI ${ }^{e}$-XIX ${ }^{e}$ siècles), Cracovie, 2010

\section{NOTES}

1. W. Schochow, Bücherschicksale. Die Verlagerungsgeschichte der Preußischen Staatsbibliothek. Berlin ; New York, 2003, p. 28-30

2. Ibid., p. 37

\section{AUTEURS}

\section{ZDZISŁAW PIETRZYK}

Directeur de la Bibliothèque Jagellonne de Cracovie 